\title{
MAGNETIC PROPERTIES OF MAGNETOTACTIC BACTERIA
}

\author{
B.M. MOSKOWITZ \\ Department of Geological and Geophysical Sciences, Princeton University, Princeton, NJ 08544, USA
}

\section{R.B. FRANKEL}

Francis Bitter National Magnet Laboratorv, Massachusetts Institute of Technologv, Cambridge, MA 02319, USA

\section{P.J. FLANDERS}

Physics Department and Laboratory for Research on the Structure of Matter, University of Pennsylvania, Philadelphia, PA 19104, USA

\author{
R.P. BLAKEMORE \\ Department of Microbiology, University of New Hampshire, Durham, NH 03824, USA
}

and

\section{B.B. SCHWARTZ}

Biomagnetech Corporation New York, NY 10017, USA

\begin{abstract}
This paper reports on the magnetic properties of magnetosomes in the freshwater magnetotactic bacterium Aquaspirillum magnetotacticum. The magnetosomes are well crystallized particles of magnetite with dimensions of 40 to $50 \mathrm{~nm}$, which are arranged within cells in a single linear chain and are within the single-magnetic-domain (SD) size range for magnetite. A variety of magnetic properties have been measured for two samples of dispersions of freeze-dried cells consisting of (1) whole cells (M-1) and (2) magnetosomes chains separated from cells (M-2). An important result is that the acquisition and demagnetization of various type of remanent magnetizations are markedly different for the two samples and suggest that remanence is substantially affected by magnetostatic interactions. Interactions are likely to be much more important in $\mathrm{M}-2$ because the extracted magnetosome chains are no longer separated from one another by the cell membrane and cytoplasm. Other experimental data for whole cells agree with predictions based on the chain of spheres model for magnetization reversal. This model is consistent with the unique linear arrangement of equidimensional particles in $A$. magnetotacticum. The magnetic properties of bacterial and synthetic magnetites are compared and the paleomagnetic implications are discussed.
\end{abstract}

\section{Introduction}

Magnetotactic bacteria in marine or freshwater aquatic sediments orient and navigate along geomagnetic field lines [1]. These bacteria are typically microaerophillic, inhabiting the microaerobic sediment between the aerobic surface and anoxic deep layers. All species of magnetotactic bacteria contain magnetosomes, which are intracytoplasmic, membrane-bound particles of magnetite, $\mathrm{Fe}_{3} \mathrm{O}_{4}$ [2]. Magnetotactic bacteria synthesize $\mathrm{Fe}_{3} \mathrm{O}_{4}$ by accumulating ferric or ferrous iron, or both, from their environment to intracellular concentration that are $10^{4}$ to $10^{5}$ times higher than ex- 
tracellular concentrations [3]. Magnetosome morphologies are species dependent [4], but are invariable within the single-magnetic-domain (SD) size range for $\mathrm{Fe}_{3} \mathrm{O}_{4}$. Magnetosomes within cells are often arranged in one or more chains with the chain axis more or less parallel to the axis of motility of the cell. Because of interparticle interactions among the magnetosomes in a chain, all the particle moments are aligned parallel to each other along the chain direction. The chain of magnetosomes thus has a permanent magnetic dipole moment which is responsible for the magnetotactic response of the organism in the geomagnetic field [5].

Magnetotactic bacteria are found in the sediments of many aquatic environments [1]. When

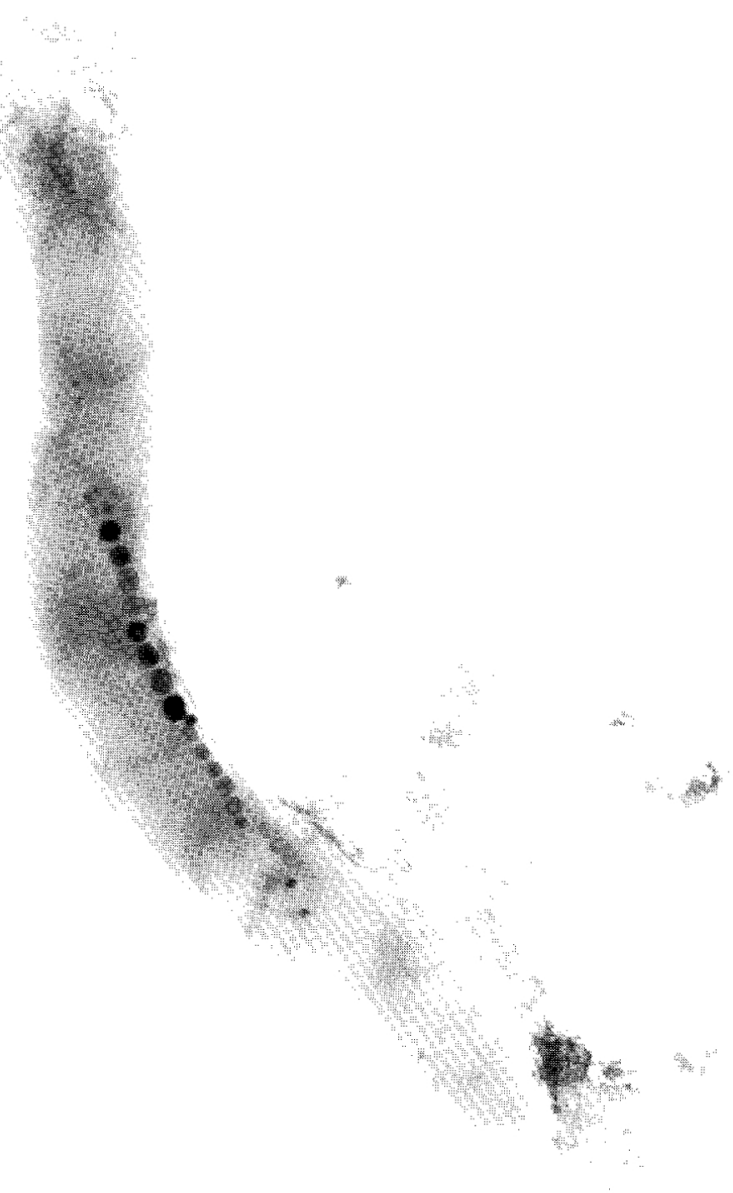


cells die, their magnetosomes or magnetosome chains could remain in the sediments, making substantial contributions to the paleomagnetic record preserved in sedimentary rocks [6]. It has been suggested that fossil magnetosomes may be the primary source of stable natural remanent magnetization in marine sediments [6-10]. Chang et al. [7] reported isolation of SD magnetite of biogenic origin from modern marine carbonate oozes and calcareous laminated sediments. They also reported isolation of $\mathrm{Fe}_{3} \mathrm{O}_{4}$ particles, with morphologies analogous to magnetosomes in current magnetotactic bacteria, in Cambrian limestones dated to 500 million years. Petersen et al. [8] have also isolated chains of SD sized particles of $\mathrm{Fe}_{3} \mathrm{O}_{4}$ with similar morphologies from deep sea sediment cores dated to 50 million years.

Aquaspirillum magnetotacticum is a freshwater magnetotactic bacterium. This organism is currently the only magnetotactic microorganism available in pure culture [11]. A. magnetotacticum contains a single chain of magnetosomes that longitudinally traverses the cell, as shown in fig. 1 . The $\mathrm{Fe}_{3} \mathrm{O}_{4}$ particles in this organism have linear dimensions of 40 to $50 \mathrm{~nm}$ and are separated from adjacent particles in the chain by approximately 4 to $10 \mathrm{~nm}$ [3]. The particles are well crystallized with truncated octahedral morphology and are oriented so that [111] faces are perpendicular to the magnetosome chain axis [4]. The number of magnetosomes per cell is variable within a population, but the average number is typically 10 to 20 magnetosomes per cell [3]. The average number of magnetosomes also varies with culture conditions, especially chelated iron concentration and dissolved oxygen tension [12]. Intact chains of magnetosomes can be separated from cell debris following cell rupture [13].

Rosenblatt et al. used static light scattering [14] and magnetically induced birefringence [15] to measure the average magnetic dipole moment per cell in suspensions of whole cells of $A$. magnetotacticum in water. Their results were consistent with estimates based on the amount of cellular $\mathrm{Fe}_{3} \mathrm{O}_{4}$ obtained from electron micrographs.

Initial bulk magnetic measurements on freezedried cells and isolated magnetosome chains of $A$. magnetotacticum were reported by Denham et al.
[16]. The saturation magnetization $\left(J_{s}\right)$ of the freeze-dried cells were consistent with an $\mathrm{Fe}_{3} \mathrm{O}_{4}$ content of about $1 \%$ dry weight of the cells. A saturation remanent magnetization $\left(J_{\mathrm{r}}\right)$ approximately equal to one-half the saturation magnetization confirmed the SD nature of the magnetosome chains. However, the coercive force $\left(H_{c}\right)$ of 21.9 $\mathrm{mT}$ was inconsistent with the Stoner-Wohlfarth (SW) model [17] for magnetization reversal by coherent rotation. Instead, Denham et al. [16] suggested that the chain of spheres or fanning model, as proposed by Jacobs and Bean [18], was a better representation of magnetization reversal along a chain of magnetosomes. In addition, the saturation magnetization on a unit weight basis was higher for the isolated magnetosomes chains whereas $J_{\mathrm{r}} / J_{\mathrm{s}}$ and $H_{\mathrm{c}}$ were lower than for the whole cells, suggesting stronger chain-chain magnetic interactions after removal of the cellular surroundings.

In this paper, we present a detailed magnetic study of magnetosomes in $A$. magnetotacticum grown in pure culture. A variety of magnetic properties have been measured on freeze-dried whole cells and magnetosomes chains separated from cells. An important result was that the acquisition and demagnetization of various type of remanent magnetizations were markedly different for the two samples and suggested that remanence was substantially affected by magnetostatic interactions. The magnetic properties of biogenic and comparably sized synthetic magnetites are also compared and discussed. In addition, hysteresis data for whole cells are shown to be consistent with predictions based on the chain of spheres model for magnetization reversal. Implications for paleomagnetism will be discussed.

\section{Experimental procedures}

\subsection{Sample preparation}

A. magnetotacticum was grown in batch culture in chemically defined medium as described previously [11]. Cells were harvested by filtration and washed in phosphate buffer. A portion of the washed cells was fixed in $1 \%$ glutaraldehyde and 
subsequently freeze-dried after removal of the fixative, or kept in suspension [12]. Cells were disrupted with a French pressure cell and the magnetic cell fraction separated in a strong magnetic field gradient. This fraction was washed and resuspended 10 times in fresh buffer, treated with $1 \mathrm{M} \mathrm{NaCl}$ and again washed several times to remove adventitious protein. Electron microscopy showed that magnetosomes in this fraction were primarily in chains. The magnetosome chains were subsequently fixed in $1 \%$ glutaraldehyde and freeze-dried or kept in suspension.

Two samples of freeze-dried materials were studied: (1) M-1, which consisted of the intact whole cells and (2) M-2, which consisted of magnetosomes chains separated from cells. Measurements of saturation magnetization were consistent with a magnetite content of about $1 \%$ dry weight of the cells for M-1 and about $14 \%$ for M-2. The freeze-dried powders were mixed in a non-magnetic matrix of epoxy for remanence measurements. It should be emphasized that because of the higher concentration of magnetite in $\mathrm{M}-2$, particle agglomeration was more likely to occur and therefore local concentrations in M-2 may be much higher than the average of $14 \%$.

\subsection{Magnetic measurements}

Hysteresis loops were measured with an ac gradient force magnetometer [19]. Samples of whole cells or extracted magnetosomes were dried onto mylar film substrates and measurements made in the plane of the film. Using the ac gradient force magnetometer, the anisotropy field distribution was determined by saturating the sample magnetically, reducing the external field to zero, rotating the sample by approximately $6^{\circ}$ for a randomly dispersed sample, or by $90^{\circ}$ for an aligned sample, and measuring the component of magnetization normal to the field $H$. The field is cycled between zero and a maximum field whose value increases on consecutive cycles [19]. The average anisotropy field $H_{\mathrm{A}}$ was determined from the initial susceptibility $\left(\chi_{0}\right)$ of a spinning sample using a novel Hall method [20]. Rotational hysteresis $W_{\mathrm{R}}$ as a function of field was measured as a sample was spun clockwise and then counter clockwise at a frequency greater than $1000 \mathrm{rpm}$ (see ref. [20] for more details). Magnetization measurements of whole cells in a water suspension before and after freezing in an applied field were made with a SQUID magnetometer.

Remanent magnetization (RM) was measured with a Schonstedt spinner magnetometer. Singleand multi-axis alternating field (af) demagnetization was conducted in a low-field environment using an af solenoid demagnetizer. For single-axis demagnetization, the axis of demagnetization was the axis along which RM was induced. Isothermal remanent magnetization (IRM) was produced using either a short-duration pulse discharge coil, with a peak field of $100 \mathrm{mT}$, or an iron-cored electromagnet, with a peak field of $800 \mathrm{mT}$. IRM acquisition curves were measured by applying incrementally increasing fields to initially demagnetized samples and noting the IRM produced. Static field demagnetization curves were measured by applying increasingly higher reverse-polarity dc fields to a saturation IRM (SIRM). Anhysteretic remanent magnetization (ARM) was imparted to the sample, initially af demagnetized at $120 \mathrm{mT}$, by applying an af of $100 \mathrm{mT}$ simultaneously with a small dc field. The af was reduced slowly to zero and the remanent magnetization recorded. All ARM's were produced by dc fields that were always parallel to the axis of the alternating field. Zero-field decay of a viscous remanent magnetization (VRM) was measured after the sample was initially af demagnetized at $120 \mathrm{mT}$ and then exposed to a constant field of $0.5 \mathrm{mT}$ for approximately $16 \mathrm{~h}$. Low-field initial susceptibility was measured using an ac susceptibility bridge.

\section{Remanence curves and coercive forces}

To facilitate comparisons among different remanent magnetization ( $R M$ ) curves and their respective average coercivities, the following notation is used. All RM curves are normalized with respect to saturation remanence. IRM acquisition and $\mathrm{dc}$ demagnetization curves are denoted by $J_{\text {ir }}(H)$ and $J_{\mathrm{d}}(H)$, respectively. The remanent coercive force, $H_{\mathrm{r}}$, is the reverse dc field necessary to reduce an initial SIRM to zero. The comple- 
ment to this is $H_{\mathrm{r}}^{\prime}$, which is the de field at which $J_{\text {ir }}(H)$ is 0.5. AF demagnetization curves of SIRM or ARM are denoted by $J_{\mathrm{ir}}(\tilde{H})$ or $J_{\mathrm{arm}}(\tilde{H})$, where $\tilde{H}$ is the peak alternating field. The median destructive field. $H_{12}$, is the af necessary to reduce an initial remanence by half. In addition, to estimate the spectral widths of the coercivity distributions exhibited by the various RM curves, let $H_{1}$ and $H_{2}$ be the fields at which the normalized intensity is, respectively, $15 \%$ and $85 \%$ of saturation (e.g., ref. [21]).

For an ensemble of non-interacting single-domain grains, Wohlfarth [22] has shown that the following relationships hold among the different RM curves:

$J_{\mathrm{d}}(H)=1-2 J_{\text {ir }}(H)$,

$J_{\text {ir }}(\tilde{H})=\frac{1}{2}\left(1+J_{\mathrm{d}}(H)\right)=d(h)$,

$J_{\text {ir }}(\tilde{H})=1-J_{\text {ir }}(H)=r(h)$.

These relations also predict that $H_{1 / 2}=H_{\mathrm{r}}^{\prime}=H_{\mathrm{r}}$ and on a plot of $J_{\text {ir }}(\tilde{H})$ and $J_{\text {ir }}(H)$, the crossover point occurs at a value $R=0.5$ [21-26].

Particle interactions, however, will tend to offset the coercivity spectra of the different RM curves, and, as a result, relations $(1 \mathrm{a}-\mathrm{c})$ will not be satisfied (e.g., refs. [21-26]). Instead, Kneller [26] proposed that the effects of particle interactions will produce two types of magnetic behavior with respect to relations $(1 \mathrm{a}-\mathrm{c})$. Type I materials are characterized by $J_{\mathrm{ir}}(\tilde{H})<d(H)<r(H), \quad R<0.5$ and $H_{1 / 2}<H_{\mathrm{r}}^{\prime}<H_{\mathrm{r}}$. In contrast, type II materials are characterized by $J_{\mathrm{ir}}(\tilde{H})>d(H) \geq r(H), R>$ 0.5 and $H_{1 / 2}>H_{\mathrm{r}}^{\prime} \geq H_{\mathrm{r}}$. Dispersed powders of SD particles, in which agglomeration of particles occur, are type I materials, whereas, SD precipitates in a non-magnetic matrix, in which minimal agglomeration occurs, are type II materials [26]. Multi-domain materials are always type I [26]. According to Kneller [26], the difference between type I and II interactions is effectively long-range interactions expressed via a mean field and shortrange, or nearest-neighbor interactions, respectively. As will be shown subsequently, the magnetic properties of M-1 and M-2 correspond to type II and type I materials, respectively.

\section{Results}

\subsection{Hysteresis measurements}

Fig. 2 shows the hysteresis curve of a random dispersion of M-1. For this sample, $J_{5}=0.6$ $\mathrm{Am}^{2} / \mathrm{Kg}, \quad H_{\mathrm{c}}=26.8 \mathrm{mT}$ and $J_{\mathrm{r}} / J_{\mathrm{s}}=0.53$. The value of $J_{5}$ was equivalent to an $\mathrm{Fe}_{3} \mathrm{O}_{4}$ content of about $1 \%$ dry weight of the cells. The remanence ratio was consistent with the theoretical value of 0.5 for a randomly oriented ensemble of uniaxial SD particles, and agrees with an earlier study by Denham et al. [16].

The anisotropy field in M-1 was determined by three different methods. First, the distribution of anisotropy fields in a randomly oriented sample of M-1 was determined as outlined above. The resulting distribution in $H_{\mathrm{A}}$ is plotted in fig. 3 (curve A) and peaks at approximately $43.8 \mathrm{mT}$. Second, this value was checked by measuring the initial susceptibility of a spinning sample with the Hall probe technique [20]. The result from this measurement was $H_{\mathrm{A}}=47.8 \mathrm{mT}$, in good agreement with the first method. Third, the anisotropy distribution for a water suspension of whole cells (M-1) dried onto a mylar film substrate in an external field of $13 \mathrm{~T}$ was determined. The anisotropy field distribution determined from this experiment (curve B, fig. 3) was nearly identical with the results obtained from the randomly dispersed sample used in the first two methods.

Hysteresis loops were also measured parallel to the direction of an orientating field. Here, a water suspension of whole cells (M-1) was dried onto

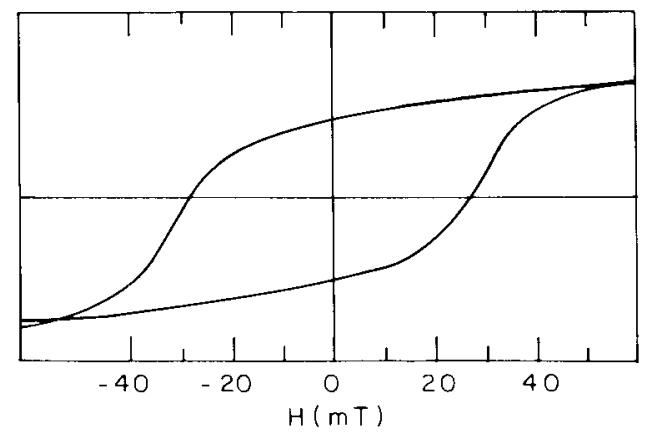

Fig. 2. Hysteresis loop for a random dispersion of frecze-dried whole cells of magnetic bacteria (M-1). 


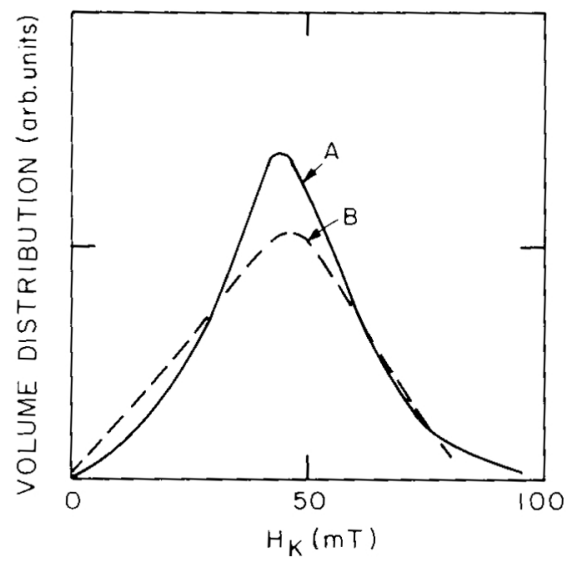

Fig. 3. Comparison of anisotropy field distribution for an aligned (curve A) and random (curve B) dispersion of freezedried whole cells. The aligned sample was produced by drying a water suspension of whole cells on a mylar film in an external field of $13 \mathrm{~T}$.

mylar film substrates in either an external field of $0.6 \mathrm{~T}$ (aligned sample) or in the earth's field (random sample) and the results of this experiment are given in table 1. Theoretically, for the state of perfect alignment, the coercive force and remanence ratio in a parallel direction are $H_{\mathrm{cil}}=$ $H_{\mathrm{A}}$ and $J_{\mathrm{r} \mid \|} / J_{\mathrm{s}}=1.0$ [17]. However, $H_{\mathrm{c} \|} \approx 0.6 H_{\mathrm{A}}$ and was approximately equal to the coercive force in the random dispersion. The latter result was consistent with the chain of spheres model [18].

Hysteresis loops and the distribution of anisotropy fields were also determined for a water suspension of extracted magnetosome chains (M-2) as a function of drying time in the earth's field. The effect of drying produces an increase in volume concentration. The results showed a dramatic decrease in the coercive force from 13.8 $\mathrm{mT}$ for a wet sample to $3.7 \mathrm{mT}$ for a completely dried sample. The distributions of $H_{\mathrm{A}}$ also shifted to lower values as the sample dried, as shown in

Table 1

Coercive forces and remanence ratios for aligned and random dispersions of whole cells

\begin{tabular}{lll}
\hline Sample & $\begin{array}{l}H_{\mathrm{c} !} \\
(\mathrm{mT})\end{array}$ & $J_{\mathrm{r} \|} / J_{\mathrm{s}}$ \\
\hline aligned & 28 & 0.85 \\
random & 28 & 0.53 \\
\hline
\end{tabular}

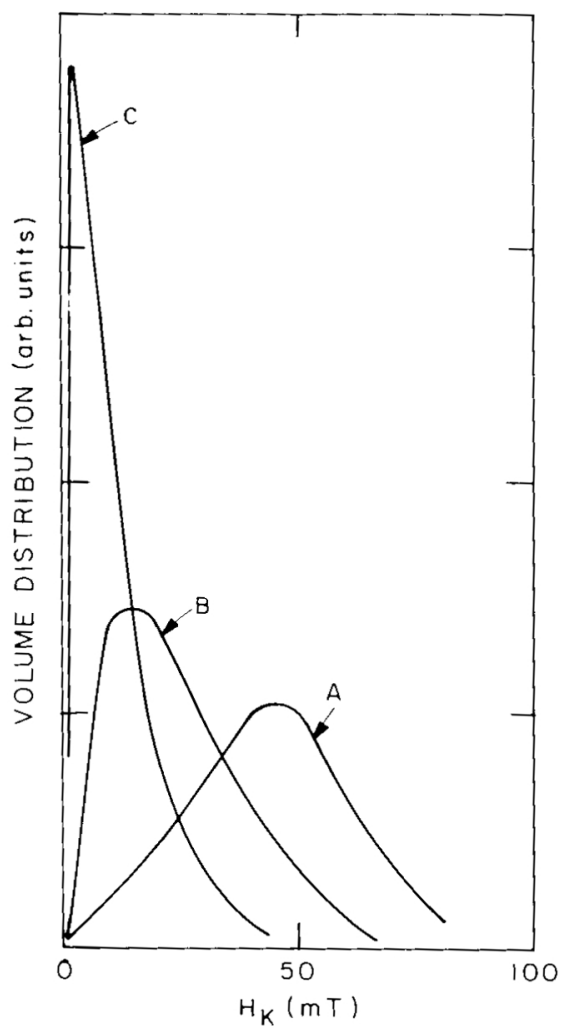

Fig. 4. Comparison of anisotropy field distributions for a random dispersion of freeze-dried whole cells (curve A) and for magnetosome chains separated from cells for samples partially dried after $30 \mathrm{~min}$ (curve B) and completely dried after 3 days (curve C)

fig. 4. Moreover, the coercive force for a completely dried sample of magnetosome chains was approximately $90 \%$ lower than the value obtained for a sample of whole cells (M-1). In contrast, $J_{\mathrm{r}} / J_{\mathrm{s}}$ decreased only slightly from 0.53 (wet) to 0.41 (dried).

Rotational hysteresis loss $W_{\mathrm{R}}(H)$ as a function of field for M-1 is shown in fig. 5. The dimensionless parameter, $R_{\mathrm{I}}=\int\left[W_{\mathrm{R}}(H) / J_{\mathrm{s}}\right] \mathrm{d} H^{-1}$, depends on the mode of magnetization reversal and provides a means to distinguish between coherent and incoherent modes [26]. From the data in fig. $5, R_{1}$ is equal to 0.92 and is 2.5 times the value predicted by the SW model but is close to the value of 1.02 that is predicted for a random assembly of particles reversing their magnetization by fanning [26]. 


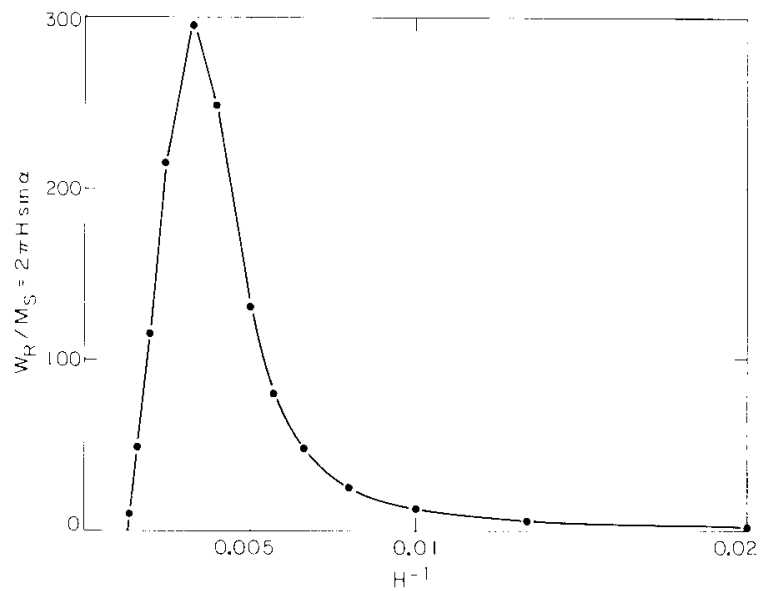

Fig. 5. Rotational hysteresis loss $W_{\mathrm{R}}$ versus $H^{-1}$ (in $\mathrm{mT}$ ) for random dispersion of freeze-dried whole cells. $W_{\mathrm{R}}$ is related to the experimental rotational lag angle $\alpha$.

Measurements of the magnetic orientation of whole cells in a water suspension at $300 \mathrm{~K}$ were made with a SQUID magnetometer. The magnetization approached saturation for fields above 1.0 $\mathrm{mT}$, as expected for an array of permanent magnetic dipoles with moments of the order of $3 \times$ $10{ }^{16} \mathrm{Am}^{2}$.

Hysteresis loops were also determined for the suspension of cells after freezing in an applied field in the SQUID magnetometer. By measuring the magnetic moment during the cooling process, it was determined that the suspension froze below $265 \mathrm{~K}$. The freezing process was found to disrupt

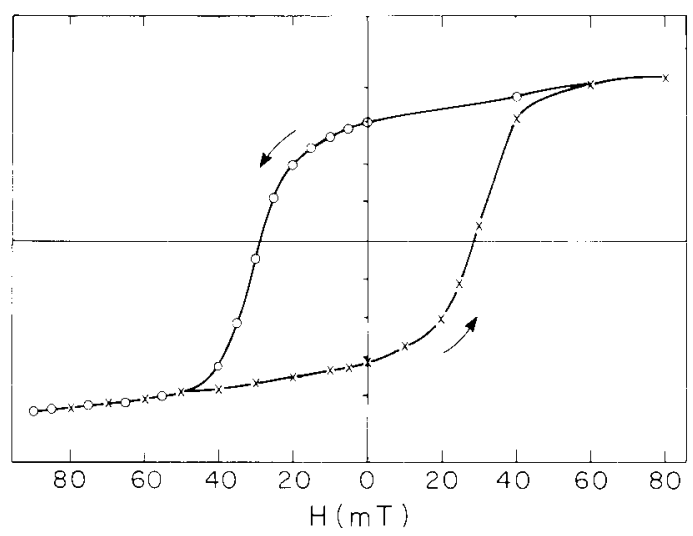

Fig. 6. Hysteresis loop for a suspension of fixed cells in water frozen in $0.9 \mathrm{~T}$ and measured in SQUID magnetometer.

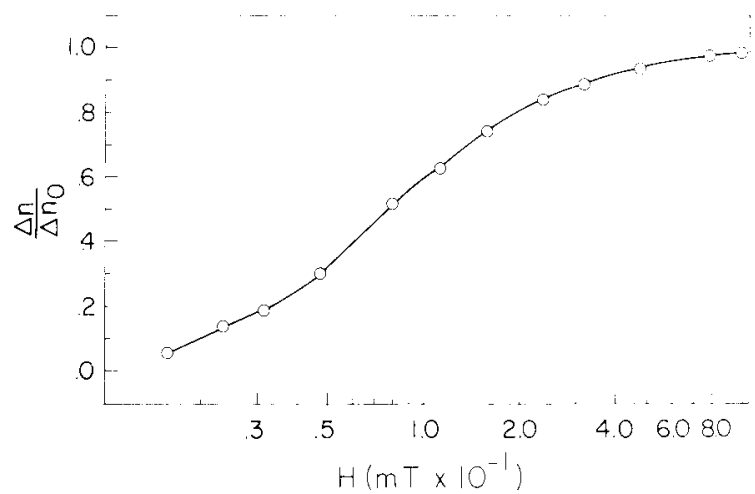

Fig. 7. Relative sample birefringence of suspension of whole cells in water as a function of applied magnetic field. The solid curve is the best fit to the data assuming an average magnetic moment per cell of $2.4 \times 10^{16} \mathrm{Am}^{2}$.

the alignment of the cells to an extent that depended on the applied field. At $115 \mathrm{mT}$. the moment decreased to $95 \%$ of the saturation value. but at $10 \mathrm{mT}$, the moment decreased to about $60 \%$ of the saturation value. The reason for this is unclear. The complete hysteresis loop for this sample is shown in fig. 6 . From this curve, $H_{c}=28$ $\mathrm{mT}$ and $J_{\mathrm{r}} / J_{\mathrm{s}}=0.91$ and agree with the results in table 1 .

\subsection{Magnetically induced birefringence}

Determination of the average magnetic dipole moment per cell was made by measuring the magnetically induced birefringence of the freeze-dried cells after resuspension in water [15]. The data in fig. 7 were fitted with an average magnetic moment per cell of $2.4 \times 10^{-16} \mathrm{Am}^{2}$. This value agrees with results from other studies [14,15]. Using an estimated volume of $\mathrm{Fe}_{3} \mathrm{O}_{4}$ per cell from electron micrographs, the average magnetic moment corresponded to about 10 magnetosomes per cell.

\subsection{Acquisition and demagnetization of IRM}

Normalized RM curves of $J_{\text {ir }}(\tilde{H})$ and $J_{\text {ir }}(H)$ for M-1 and M-2 are shown in fig. 8 and coercivities and spectral parameters are listed in table 2. Both samples saturated by $60 \mathrm{mT}$, but M-1 had a slightly narrower coercivity spectrum for IRM acquisition (table 2) and approached saturation at 


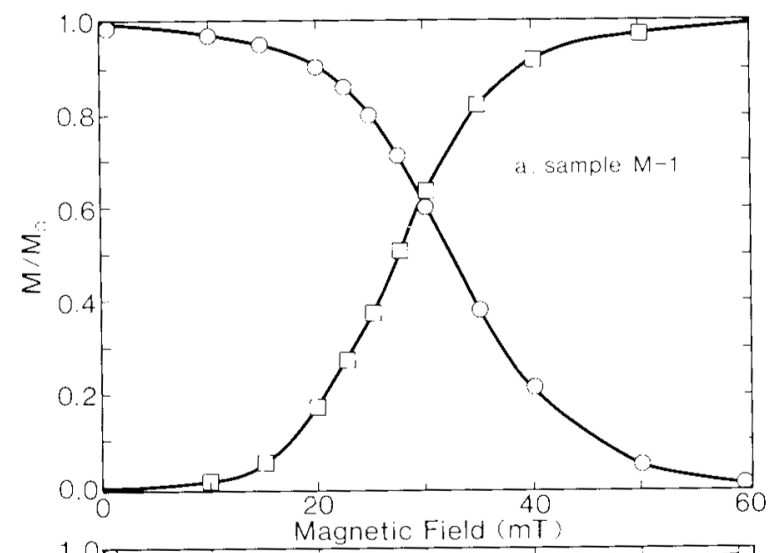

a slightly faster rate than $\mathrm{M}-2$. In contrast, there were marked differences between samples during the demagnetization of SIRM, as illustrated in fig. 8. M-2 exhibited a wider spectral width $\left(\tilde{H}_{1} / \tilde{H}_{2}\right.$ $=0.17$ ), SIRM decayed more rapidly with $\tilde{H}, R$ was equal to 0.21 , complete demagnetization occurred at approximately $30 \mathrm{mT}$, and the af demagnetization spectrum was offset towards lower fields with respect to its do magnetization spectrum. For M-1, however, the spectral width was narrower $\left(\tilde{H}_{1} / \tilde{H}_{2}=0.54\right), R$ was equal to 0.62 . complete demagnetization occurred at approximately $60 \mathrm{mT}$, and the af demagnetization

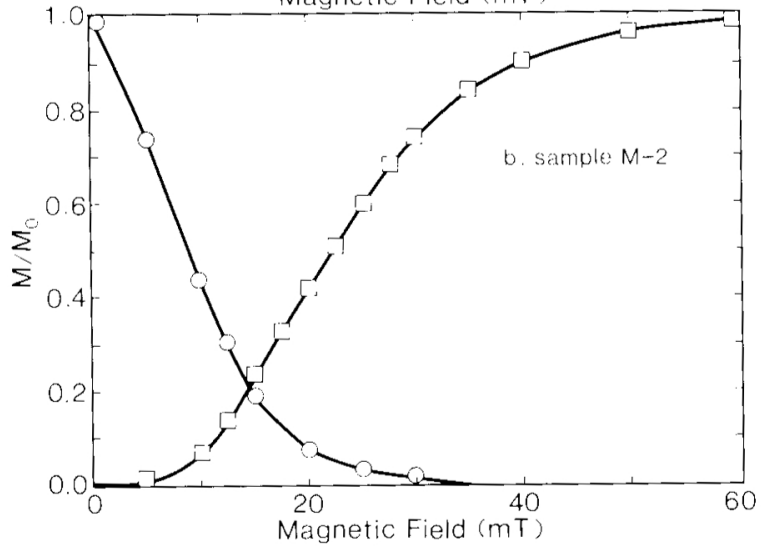

Fig. 8. Normalized curves of acquisition and af demagnetization of SIRM for (a) freeze-dried whole cells (M-1) and (b) freeze-dried magnetosome chains separated from cells (M-2).

Table 2

Coercivities and coercivity distribution parameters for whole and separated magnetosomes

\begin{tabular}{lcc}
\hline $\begin{array}{l}\text { Coercivity } \\
(\mathrm{mT})\end{array}$ & $\mathrm{M}-1$ & $\mathrm{M}-2$ \\
\hline$H_{\mathrm{c}}$ & 26.7 & 3.7 \\
$H_{\mathrm{r}}$ & 27.6 & 16.6 \\
$H_{\mathrm{r}}^{\prime}$ & 27.5 & 22.4 \\
$H_{1 / 2 \mathrm{irm}}$ & 32.2 & 9.0 \\
$H_{1 / 2 \mathrm{arm}}$ & 33.7 & 12.3 \\
$\left(H_{1} / H_{2}\right)_{\mathrm{irm}}{ }^{\mathrm{a})}$ & 0.53 & 0.36 \\
$\left(H_{1} / H_{2}\right)_{\mathrm{irm}}$ & 0.50 & 0.19 \\
$\left(H_{1} / H_{2}\right)_{\mathrm{irm}}{ }^{\mathrm{d}}$ & 0.54 & 0.17 \\
$\left(H_{1} / H_{2}\right)_{\mathrm{arm}}$ & 0.55 & 0.30 \\
\hline
\end{tabular}

a) Distribution parameters for IRM acquisition.

h) Distribution parameters for de demagnetization of SIRM.

c) Distribution parameters for af demagnetization of SIRM.

(1) Distribution parameters for af demagnetization of $0.1 \mathrm{mT}$ ARM.
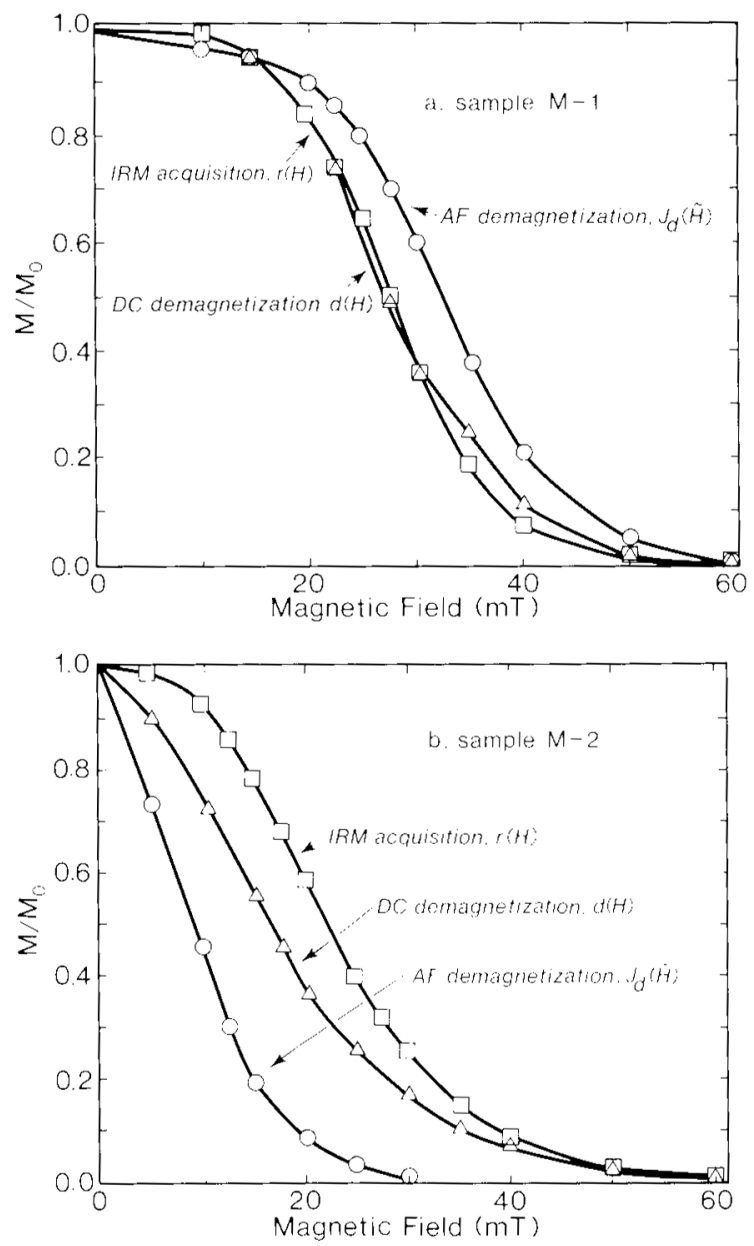

Fig. 9. Normalized remanence curves as a function of the applied field for (a) M-1 and (b) M-2. For comparison, all curves are plotted in terms of the af demagnetization curve according to the Wohlfarth relationships [eq. (1) of text]. 
spectrum was offset towards higher fields with respect to its de magnetization spectrum. The various estimates of coercivities were also significantly different between the two samples (see table 2). Ratios of $H_{1 / 2} / H_{\mathrm{r}}$ and $H_{\mathrm{r}}^{\prime} / H_{\mathrm{r}}$ were 1.17 and 1.00 for M-1 and 0.54 and 1.35 for M-2.

The difference in demagnetization characteristics between M-1 and M-2 was demonstrated further when remanence data were plotted in terms of $J_{\mathrm{ir}}(\tilde{H})$, according to relations $(1 \mathrm{a}-\mathrm{c})$. The results are shown in fig. 9 and confirmed that M-1 exhibited type II behavior, whereas M-2 exhibited type I behavior using the classification scheme of Kneller [26]. The results also illustrated that the most efficient method for erasing an SIRM was af demagnetization in M-2 and de demagnetization in M-1. It should be noted, however, that when multi-axis af demagnetization was employed, it was found to be more efficient than either singleaxis af or dc demagnetization methods. Furthermore, the $R$ parameter determined from multi-axis af demagnetization of SIRM was lower than its value obtained from single-axis demagnetization [23].

\subsection{Anhysteretic remanent magnetization}

Fig. 10 compare ARM induction curves for M-1 and M-2. The approach to saturation was much more rapid in $\mathrm{M}-1$ and $\mathrm{M}-2$. For example, the ARM of M-1 was approximately $70 \%$ of saturation in a field of only $0.6 \mathrm{mT}$, whereas M-2

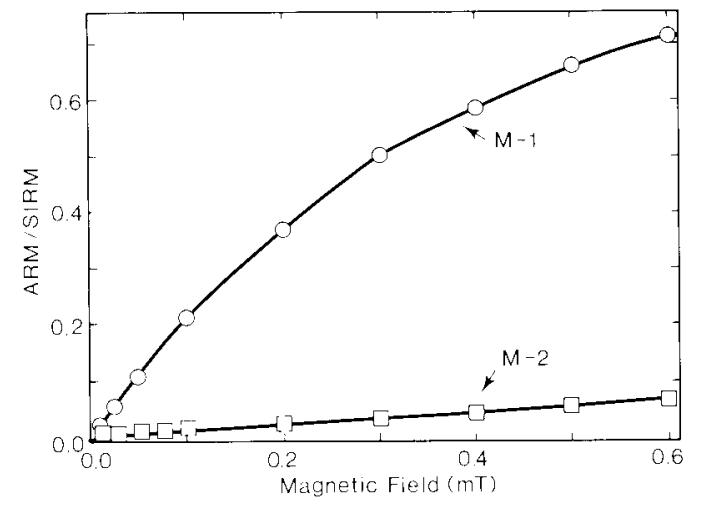

Fig. 10. Normalized acquisition of anhysteretic remanent magnetization as a function of applied dc field for M-1 and M-2.

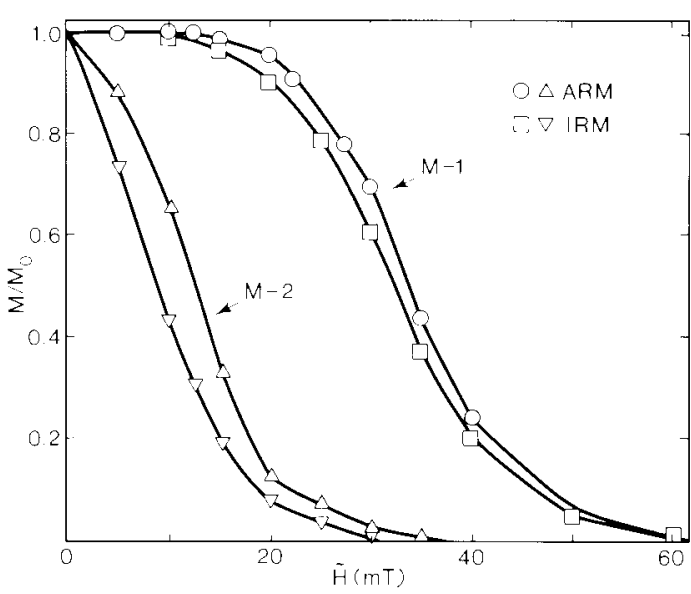

Fig. 11. Normalized af demagnetization curves of ARM and SIRM. ARM was acquired in de ficld of $0.1 \mathrm{mT}$.

was barely at $10 \%$ of saturation in the same field. As a comparison, for comparable sized dispersed single-domain powders, ARM was less than $30 \%$ of saturation by $0.6 \mathrm{mT}[27,28]$. Initial anhysteretic susceptibilities (actually $\chi_{\mathrm{arm}} / \mathrm{SIRM}$ ) were $2.68(\mathrm{kA} / \mathrm{m})^{-1}$ and $0.13(\mathrm{kA} / \mathrm{m})^{-1}$ for $\mathrm{M}-1$ and $\mathrm{M}-2$, respectively.

\subsection{Lowrie-Fuller test}

A commonly used procedure in rock magnetism is the Lowrie-Fuller test [29,30], in which the af demagnetization spectra of a strong-field remanence, such as SIRM, is compared to a weak-field remanence, such as ARM. This test has been shown to discriminate between SD-like and MD-like particles. For example, weak-field ARM in SD and small MD particles exhibit more resistance to af demagnetization than strong-field IRM, whereas large MD particles exhibit the opposite behavior (see ref. [30] for a complete discussion).

The results of the Lowrie-Fuller test for M-1 and $\mathrm{M}-2$ are shown in fig. 11 and predicted that the domain state was $\mathrm{SD}$, as one would have suspected based on particle size alone. The ARM and SIRM demagnetization curves were also similar in form for each sample but quite different between samples. The initial plateaus in the ARM and SIRM curves in $M-1$, which were missing in M-2, indicated that a threshold field must be 
reached before demagnetization started. In contrast, the rapid demagnetization of ARM and SIRM in $\mathrm{M}-2$, reminiscent of demagnetization curves for multi-domain materials, suggested that moments with very low coercivities were present. Variable and strong interaction fields in M-2 may be responsible for its low stability to af demagnetization. The results of fig. 11 also demonstrated that interactions in SD materials apparently do not effect the outcome of the Lowrie-Fuller test and agree with results obtained by Cisowski [23].

The ARM and SIRM data can be compared directly to results given in ref. [8], which were obtained from deep-sea sediments that possibly contained biogenic magnetite. Petersen et al. [8] used the parameters $\Delta \mathrm{LF}=H_{1 / 2 \mathrm{arm}}-H_{1 / 2 \mathrm{irm}}$. ARM/SIRM, and $H_{1 / 2 \text { irm }}$ to distinguish between biogenic SD and inorganic MD magnetite. The magnetic properties of some of their samples, which were later found to contain magnetite particles with morphologies that suggested a biogenic origin, fell within a narrow range of values (their A component) with $\Delta \mathrm{LF} \approx 4.5-7.0 \mathrm{mT}$, ARM/ SIRM $\approx 0.07-0.10$ and $H_{1 / 2 \mathrm{irm}} \approx 12-15 \mathrm{mT}$. By contrast, our magnetic results on biogenic magnetite were significantly different from those attributed to their A component; specifically, $\Delta \mathrm{LF}$ $=1.5 \mathrm{mT}, \mathrm{ARM} / \mathrm{SIRM}=0.11$ and $H_{1 / 2 \mathrm{irm}}=32.2$ $\mathrm{mT}$ for M-1 and $\Delta \mathrm{LF}=3.3, \mathrm{ARM} / \mathrm{SIRM}=0.005$ and $H_{1 / 2 \mathrm{irm}}=9.0 \mathrm{mT}$ for $\mathrm{M}-2$.

\subsection{Viscous remanent magnetization}

There were also significant differences in VRM behavior between $\mathrm{M}-1$ and $\mathrm{M}-2$. The viscous moments normalized to SIRM, acquired in a steady field of $0.5 \mathrm{mT}$ after $t_{\mathrm{a}}=16 \mathrm{~h}$, were approximately $0.08 \%$ for M-1 and $0.76 \%$ for M-2. The zero field decay of VRM is shown in fig. 12 The experimental decay curves were fitted to polynomial functions in $\ln t$ by step-wise regression. For decay times $t_{\mathrm{d}}$ less than $t_{\mathrm{a}}, \mathrm{M}-1$ exhibited a linear logarithmic decay $(V R M \propto \ln t)$. In contrast, the best fit polynomial for $\mathrm{M}-2$ consisted of a constant term plus a term proportional to $(\ln t)^{3}$. After 16 $\mathrm{h}\left(t_{\mathrm{d}}>t_{\mathrm{a}}\right)$, the decay curves started to tail off but with a significant fraction $(40-50 \%)$ of the original VRM remaining after a decay time equal to the

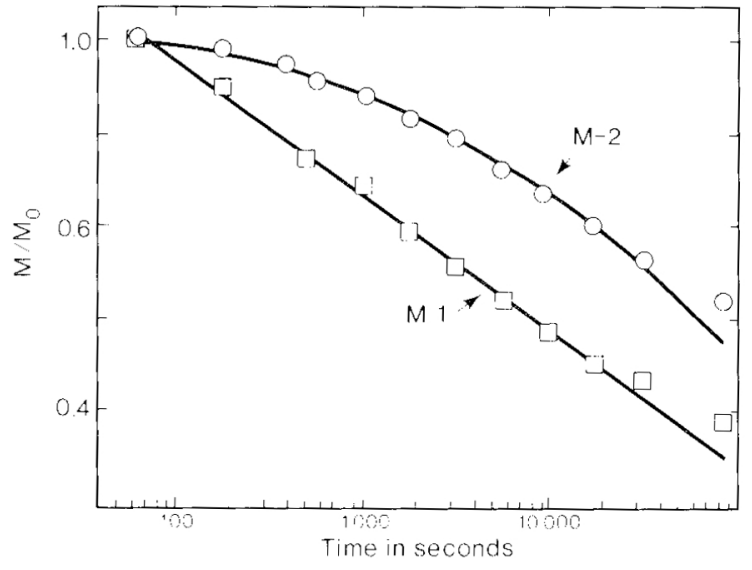

Fig. 12. Normalized zero-field decay of VRM. Solid lines are best fit polynomials in $\ln t$ to the data.

original exposure time, this effect being more pronounced in M-2. In other words, acquisition of VRM proceeded more rapidly than its subsequent decay.

\section{Discussion}

\subsection{Magnetostatic interactions}

Magnetostatic interactions are most likely responsible for the marked contrast between the magnetization and demagnetization characteristics of $\mathrm{M}-1$ and $\mathrm{M}-2$. Stronger particle interactions are assumed to correspond to greater degree of particle agglomerations. Particle agglomeration is much more likely to occur in $\mathrm{M}-2$ because the extracted magnetosome chains are no longer separated by the cell membranes and cytoplasm of the bacteria and, hence, are closer together on average. In this case, the interaction fields make it more difficult to magnetize than to demagnetize a sample (type I behavior). The offsets between the coercivity spectra, as observed in $\mathrm{M}-2$, also have been observed in rocks, dispersed magnetic powders and magnetic recording media $[21,23,25]$. In contrast, the individual chains are still intact within the bacteria in M-1 and are therefore separated from one another by the cell membranes and cytoplasm. Thus, agglomeration of the chains should be reduced considerably. In this case, the 
af spectra are shifted towards higher fields and the de spectra are shifted towards lower fields. The apparent reduction in agglomeration is apparently sufficient to produce type II behavior, but interactions must still be important in M-1; otherwise, the RM curves should be described exactly by the Wohlfarth relations. This type of behavior has not been reported in samples consisting of dispersed powders, although it has been observed in certain precipitation alloys [26].

It is well known that magnetic interactions are responsible for ARM properties [26]. An estimate of the magnitude of the interaction fields in $\mathrm{M}-1$ and $\mathrm{M}-2$ can be obtained from the ARM results following Jaep [31,32]. Strictly speaking, Jaep's theory is valid only for interactions that are longrange and can be modeled by a mean field approximation [33]; however, adjusting the theory to take into account short-range interactions results in a similar equation of ARM [33]. For the region where the intensity of ARM is linearly dependent on the external field $h_{\mathrm{dc}}$, the ARM in an ensemble of interacting single-domain grains is approximately,

$\mathrm{ARM} / \mathrm{SIRM}=B h_{\mathrm{dc}} /\left(k T / J_{\mathrm{s}}+\lambda\right)$,

where $h_{\mathrm{dc}}$ is the applied field, $\lambda$ is the interaction field, $B \approx J_{\mathrm{sb}} / J_{\mathrm{s}}\left(T / T_{\mathrm{b}}\right)^{1 / 2}$, and the subscript " $\mathrm{b}$ " refers to the value of the parameter at the blocking temperature [31.32]. However, the experimental blocking temperature and, subsequently $B$ and $\lambda$, for the bacterial magnetites were not determined directly because of the possible adverse chemical changes which could be induced by heating the sample close to its Curie temperature. Nevertheless, estimates of $\lambda$ can still be obtained from estimates of $B$ by using reasonable limits for $T_{\mathrm{b}}$ as follows.

The parameter $B$ was calculated for different values of $T_{\mathrm{b}}$ at $5^{\circ}$ intervals between 500 and $575^{\circ} \mathrm{C}$ with $J_{\mathrm{s}}$ taken from Pauthenet [34]. $\lambda$ was then determined using eq. (2) and the initial slope of the ARM induction curve (fig. 10). Using the limits for $T_{\mathrm{b}}, \lambda$ was found to very between 0.012 and $0.065 \mathrm{mT}$ for M-1, and between 0.98 and 2.4 $\mathrm{mT}$ for $\mathrm{M}-2$ and indicated that the interaction fields were approximately 50 times greater in $\mathrm{M}-2$.
In addition, an average distance between chains of particles (assuming an average chain length of 10 particles) that would be necessary to produce a field equal to $\lambda$ was also determined. This calculation predicted that the particle chains were 1-3 $\mu \mathrm{m}$ apart in $\mathrm{M}-1$, but only $0.3-0.4 \mu \mathrm{m}$ apart in M-2. The former estimate was consistent with the average size of an individual bacterium.

The time dependence of magnetization can also be related qualitatively to the degree of particle interactions. For example, most theories of magnetic viscosity for non-interacting SD particles predict a linear $\ln t$ dependence of magnetization [35]. However, the time dependence commonly observed is non-linear in $\ln t$ for many rocks. dilute fine-particle dispersions and spin glasses (e.g., refs. [36-40]). Using a mean random field approach, Walton and Dunlop [39] predicted that the aquisition and decay of VRM should follow a polynomial ln $t$ dependence. As our ARM results suggested, interactions fields were much lower in M-1, which exhibited a linear $\ln t$ dependence. than in M-2, which did not. The distinct curvature exhibited by the time dependence of magnetization of M-2 has also been observed in synthetic SD and small MD magnetites [36].

However, to explain the observation that acquisition of VRM is faster than the corresponding decay, a different distribution of activation times must be involved during acquisition and decay. It is possible that a distribution of interactions fields could produce the asymmetry between acquisition in an external field and decay in zero field. This asymmetry has also been observed in many other SD and MD materials (e.g., refs. $[36,37])$; however, there is not yet a satisfactory theoretical explanation for this observation.

\subsection{Bacterial versus synthetic magnetite}

The results of our experiments also offer an excellent opportunity to compare and contrast the magnetic properties of bacterial magnetite to comparably size synthetic magnetite, particularly concerning the role of magnetostatic interactions. The importance of interactions is dispersed magnetic powders has been suggested often (e.g. refs. $[21,23,26])$. Invariably, for samples consisting of 
dilute dispersions of magnetite in a non-magnetic matrix, particle interactions are prevalent due to particle agglomerations. Moreover, most synthetic magnetites are compared of a distribution of particle sizes. The combined effects of magnetostatic interactions and a distribution of particle sizes play an important role in determining the magnetic properties of an ensemble of SD particles. The separation of these two effects is particularly important for various methods of magnetic granulometry, which are being used in many studies of the environmental applications of magnetism (e.g., ref. [41]). The narrow particle size distribution of the bacterial magnetite effectively removes particle size as a variable; hence, differences in magnetic properties between the freeze-dried samples should be due to interactions alone.

Magnetic properties of synthetic magnetites have been taken from the rock magnetic literature [21,42-45] and included: (1) chemically precipitated equidimensional particles, with grain sizes ranging from 25 to $220 \mathrm{~nm}$; and (2) acicular particles with axial ratios of $8: 1$ and $7: 1$ and absolute dimensions of $30 \times 200 \mathrm{~nm}^{2}$ and $40 \times$ $3500 \mathrm{~m}^{2}$, respectively. The acicular particles have similar grain dimensions to the magnetosome chains in M-1. These samples were chosen for

Table 3

Magnetic parameters of bacterial and synthetic magnetite

\begin{tabular}{|c|c|c|c|c|}
\hline Parameter & M-1 & $M-2$ & Cubic ${ }^{a)}$ & Acicular ${ }^{b}$ \\
\hline$\chi_{\mathrm{arm}} / \chi_{0}$ & 148.7 & 1.46 & $\begin{array}{l}5.19- \\
9.76\end{array}$ & $\begin{array}{l}5.98- \\
38.2\end{array}$ \\
\hline $\begin{array}{l}\chi_{\mathrm{arm}} / \mathrm{SIRM} \\
\left(1 / \mathrm{kAm}^{-1}\right)\end{array}$ & 2.675 & 0.125 & $\begin{array}{l}0.150_{-} \\
0.188\end{array}$ & 0.113 \\
\hline $\begin{array}{r}\chi_{0} / J_{\mathrm{s}}\left(\times 10^{-3}\right. \\
\left.\left(1 / \mathrm{kAm}^{-1}\right)\right)\end{array}$ & 9.86 & 34.88 & 8.75 & 6.87 \\
\hline $\begin{array}{l}\mathrm{SIRM} / \chi_{0} \\
\quad(\mathrm{kAm}-1)\end{array}$ & 55.58 & 11.73 & $\begin{array}{l}32.08- \\
51.68\end{array}$ & 53.12 \\
\hline $\begin{array}{l}S_{\mathrm{d}} / J_{\mathrm{s}} \\
\quad\left(\times 10^{-4}\right)\end{array}$ & 0.26 & 0.92 & 0.60 & - \\
\hline$J_{\mathrm{r}} J_{\mathrm{s}}$ & 0.53 & 0.41 & 0.28 & $\begin{array}{l}0.36- \\
0.45\end{array}$ \\
\hline$H_{\mathrm{r}} / H_{\mathrm{c}}$ & 1.02 & 4.49 & $1-2$ & $1-2$ \\
\hline
\end{tabular}

a) Values were interpolated for a grain size of $42 \mathrm{~nm}$. Results were taken from refs. [42-45].

b) Acicular magnetites were $30 \times 200 \mathrm{~nm}^{2}$ [45] and $40 \times 350$ $\mathrm{nm}^{2}$ (King, unpublished).
Table 4

Coercivities of bacterial and synthetic magnetites

\begin{tabular}{lrrrl}
\hline $\begin{array}{l}\text { Coercivity } \\
(\mathrm{mT})\end{array}$ & $\overline{\mathrm{M}}-1$ & $\mathrm{M}-2$ & Cubic $^{\mathrm{a})}$ & Acicular $^{\mathrm{b})}$ \\
\hline$H_{\mathrm{c}}$ & 26.7 & 3.7 & 21.3 & $30.5-43.8$ \\
$H_{\mathrm{r}}$ & 27.6 & 16.6 & 38.4 & $50.5-60.0$ \\
$H_{\mathrm{r}}^{\prime}$ & 27.5 & 22.4 & 50.6 & 67.7 \\
$H_{1 / 2 \mathrm{irm}}$ & 32.2 & 9.0 & 28.3 & 43.4 \\
$H_{1 / 2 \mathrm{arm}}$ & 33.7 & 12.3 & - & 57.1 \\
\hline
\end{tabular}

a) Values were interpolated for a grain size of $42 \mathrm{~nm}$. Results were taken from ref. [21].

b) Acicular magnetites were $30 \times 200 \mathrm{~nm}^{2}$ [21] and $40 \times 350$ $\mathrm{nm}^{2}$ [44].

comparison because their ARM, SIRM, $\chi_{0}, S_{\mathrm{d}}$ and coercivity data were available in the literature. Additionally, to eliminate errors arising from uncertainties in the concentration of magnetite in our samples, ratios of magnetic parameters that are independent of concentration were used for comparisons. Parameter ratios are summarized in table 3 and coercivity data are summarized in table 4.

\subsubsection{Parameter ratios}

Particle interactions should have a pronounced effect on the ratios $\chi_{\text {arm }} / \chi_{0}$ and $\chi_{\text {arm }} /$ SIRM with $\chi_{\mathrm{arm}}$ decreasing and $\chi_{0}$ increasing with the strength of the interaction field [26]. The ratios $\chi_{\text {arm }} / \chi_{0}$ and $\chi_{\text {arm }} /$ SIRM for M-2 were similar to, but slightly lower than, those for the equant magnetites. On the other hand, the same ratios for M-1 were at least 10 times higher than the equant magnetites and 4-10 times higher than the acicular magnetites. The similar ARM results between M-2 and the synthetic magnetites suggest that similar interactions, presumably due to agglomeration effects are responsible for ARM in both types of materials. This conclusion is supported further by the observation that all synthetic dispersed magnetites exhibit type I behavior $[21,23]$, just like M-2. Significantly, it is interesting to note that the volume percent of magnetite in M-2 is higher than the dispersed synthetic powders and suggests that dilution alone does not reduce agglomeration. The higher values of $\chi_{\mathrm{arm}} / \chi_{0}$ and $\chi_{\text {arm }} /$ SIRM for M-1, undoubtedly related to the reduced effects of agglomerations, indicate that acicular magnetite is not a good analog for ARM 
in M-1, even though the particle dimensions are approximately the same.

The $\chi_{0} / J_{\mathrm{s}}$ and SIRM $/ \chi_{0}$ parameters for M-1 were at the high end of the range of values reported for synthetic magnetites (table 3). By contrast, for M-2, $\chi_{0} / J_{\mathrm{s}}$ was higher and SIRM $/ \chi_{0}$ lower than the values in $\mathrm{M}-1$ and the synthetic magnetites. The effects of particle agglomerations on $\chi_{0}$ are probably responsible for these differences because the effects of agglomerations on the intensity of SIRM appear to be minor, as evidenced by the smaller decrease in $J_{\mathrm{r}} / J_{\mathrm{s}}$ between M-1 and M-2 (table 3). Particle agglomerations can produce an increase in $\chi_{0}$ by effectively producing a general decrease in shape anisotropy in a dispersion of particles. The shift ir the anisotropy field distribution toward lower fields with drying time (or equivalently with increasing agglomeration, see fig. 4) is consistent with a decrease in shape anisotropy.

The ratio $S_{\mathrm{d}} / J_{\mathrm{s}}$ for the bacterial and synthetic magnetites, reduced to $h=0.1 \mathrm{mT}$, is shown in table 3 . The trend in this ratio, $\mathrm{M}-2>$ equant magnetite $>\mathrm{M}-1$, corresponds to the increase in the volume concentration of magnetite in each sample and suggests that increased particle interactions also increases the viscosity coefficient.

\subsubsection{Coercivity}

Values of coercivities for bacterial and synthetic magnetites are summarized in table 4 . For M-1, $H_{\mathrm{c}}$ and $H_{1 / 2}$ were higher and $H_{\mathrm{r}}$ and $H_{\mathrm{r}}^{\prime}$ lower than they were for equant magnetites and reflected the difference between the interaction fields in type I (synthetic equant magnetite) and type II (M-1) materials. Dankers [24] observed that in weakly magnetic SD hematite, where interactions should be negligible, $H_{\mathrm{r}}^{\prime} \approx H_{\mathrm{r}}$, in agreement with the results for $\mathrm{M}-1$. In contrast, $\mathrm{M}-2$ had $H_{\mathrm{r}}^{\prime}>H_{\mathrm{r}}$, as well as much lower coercivities than observed in the synthetic magnetites, presumably due to the increased in particle interactions in M-2. However, Dunlop [45] observed only minor changes in coercivity in nearly $\mathrm{SD}$ sized magnetites with concentrations up to $30 \%$ by volume.

Whereas the absolute values of coercivities between M-2 and the synthetic magnetites are differ- ent, the ratios $H_{1 / 2} / H_{\mathrm{r}}$ and $H_{\mathrm{r}}^{\prime} / H_{4}$ are not. For example, Dunlop [21] found that $H_{\mathrm{r}}^{\prime} / H_{\mathrm{r}}=$ $1.25-1.38$ and $H_{1 / 2} / H_{\mathrm{r}}=0.62-0.86$ for dispersed SD magnetites. In comparison, in $\mathrm{M}-2, H_{\mathrm{r}}^{\prime} / H_{\mathrm{r}}=$ 1.35 and $H_{1 / 2} / H_{\mathrm{r}}=0.54$. However, the similar value of these ratios for the two sets of samples is only an expression of the more general relationship, $H_{\mathrm{r}}^{\prime}+H_{1 / 2} \approx 2 H_{\mathrm{r}}$, which was found to hold for dispersed samples of magnetite ranging in size from 0.1 to $250 \mu \mathrm{m}[21,24]$. According to Kneller [26], $H_{\mathrm{r}}^{\prime}+H_{1 / 2}=2 H_{\mathrm{r}}$ is predicted for interacting type I SD materials, in which interactions can be modeled by a mean field approximation. However, M-1, a type II material, also approximately obeys this relationship, so its significance is unclear.

It is also interesting to compare the ratio $H_{\mathrm{r}} / H_{c}$ between sample M-1 and M-2 (table 3). Theoretically, in a randomly oriented ensemble of coherently reversing SD particles, $1<H_{\mathrm{r}} / H_{\mathrm{c}}<2$ [26]. The data for M-1 and the synthetic magnetites agree with the predicted SD values. However, $H_{\mathrm{r}} / H_{\mathrm{c}}$ for M-2 is significantly higher than predicted. The high value of $H_{\mathrm{r}} / H_{\mathrm{c}}$ is due to the much greater decrease of $H_{c}$ when the magnetosomes are extracted from the cells. $H_{c}$ decreases nearly $90 \%$ but $H_{\mathrm{r}}$ decreases only $40 \%$ upon extraction. High values of $H_{\mathrm{r}} / H_{\mathrm{c}}$ often indicate the presence of SP particles or a mixture of soft and hard coercivity components [26], although in our case SP particles seem unlikely. On the other hand, interactions may either produce low coercivity components or produce an increase in susceptibility such that $J_{\mathrm{r}}$ is balanced only by the induced magnetization $-\chi_{0} H_{c}$ [21]. The af demagnetization of SIRM and ARM for M-2, however, is consistent with low coercivity moments.

\subsection{Chain-of-spheres model}

The SW model for coherent rotation of magnetization due to shape anisotropy predicts that the coercive force in M-1 should be approximately $140 \mathrm{mT}$ for an axial ratio of 10:1 (i.e., a chain length of 10 particles). The much lower observed coercive force of $23.8 \mathrm{mT}$ in $\mathrm{M}-1$ suggests a noncoherent reversal mechanism. A likely mechanism for moment reversal along a chain of magneto- 
somes is the fanning or chain of spheres model [18], as first suggested by Denham et al. [16].

The chain-of-spheres model was originally developed to explain the coercive force in elongated single domain grains [18]. This model should be ideal to describe the reversal mode in M-1 because of the unique linear arrangement of equidimensional particles in bacterium. Experimental values for the rotational hysteresis integral and the ratio of coercive forces for random and aligned sample for M-1 are compared with predictions based on the SW and chain-of-spheres models in table 5. The experimental data are in excellent agreement with the chain-of-spheres model.

In the original chain-of-spheres calculations [18], however, the spheres were assumed to be touching. It is clear from electron micrographs that this is not the case for magnetosomes in magnetotactic bacteria. To account for a finite separation distance, the model coercive forces must be reduced by $(1+\beta)^{-3}$, where $\beta=x / a, a$ is the particle diameter and $x$ is the separation distance. This correction assumes that $\beta$ is a constant along the chain length in an individual bacterium. Coercive force for randomly oriented chains as a function of $\beta$ are plotted in fig. 13 for three different reversal models [18]: (1) symmetric fanning (model A), where the magnitude of the angle of fanning is constant along the length of the chain; (2) non-symmetric fanning (model $A^{\prime}$ ), where the angle of fanning is not constant; and (3) parallel rotation (model $\mathrm{B}$ ). The results of these calculations are as follows.

First, $H_{c}$ increases continuously with chain length for both models $\mathrm{A}$ and $\mathrm{B}$, whereas, in model $\mathrm{A}^{\prime}, H_{\mathrm{c}}$ is nearly independent of chain length for $n$ greater than 6 [18]. Second, the values for $\beta$ estimated from the observed coercive force for

Table 5

Calculated and observed valucs for selected magnetic parameters. Model values are based on an ensemble of random uniaxial particles

\begin{tabular}{llll}
\hline Parameter & $\begin{array}{l}\text { SW } \\
\text { model }\end{array}$ & $\begin{array}{l}\text { Fanning } \\
\text { model }\end{array}$ & M-1 \\
\hline$R_{\mathrm{I}}$ & 0.380 & 1.02 & 0.92 \\
$H_{\mathrm{c} \text { (random) } / H_{\mathrm{c}} \text { (aligned) }}$ & 0.479 & $1.08-1.13$ & 0.93 \\
\hline
\end{tabular}

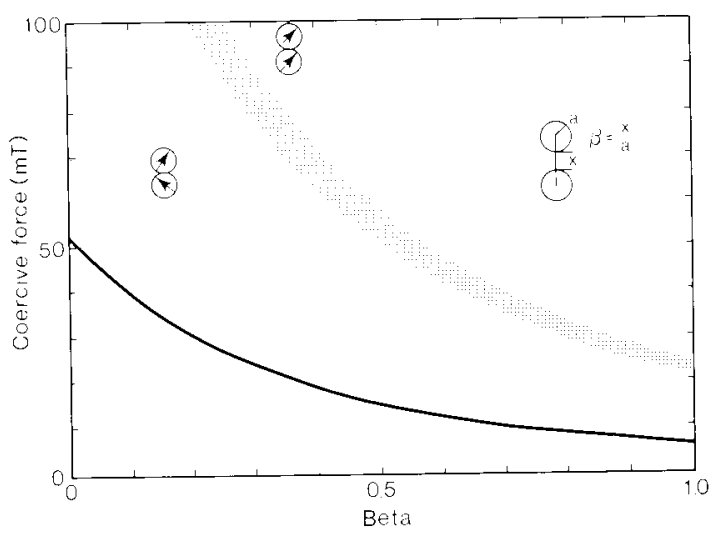

Fig. 13. Coercive forces for a randomly oriented chain of spheres as a function of sphere separation $(\beta)$. Model calculations are based on the the chain of spheres model [18]. The dark stipple curve is for parallel rotation (model B), the light stipple curve is for symmetric fanning (model A), and the solid curve is for non-symmetric fanning (model $A^{\prime}$ ). The width of each curve takes into account different chain lengths from 6 to an infinite number of spheres. See text for further details.

M-1 $\left(H_{\mathrm{c}}=26.7 \mathrm{mT}\right)$ using fig. 13 are (1) 0.25 for model $A^{\prime}$, (2) $0.37-0.46$ for model $A$ and (3) $0.8-0.9$ for model B. Models $A$ and $A^{\prime}$ predict values of $\beta$ that are consistent with observation $(x=3-18 \mathrm{~nm}, \beta \approx 0.07-0.43$ [3]). In contrast, model $\mathrm{B}$ predicts values of $\beta$ that are too high and therefore it seems unlikely that coherent rotation is important. Third, to account for the observed coercivity spectrum (i.e., $H_{1} / H_{2} \neq 1$, see table 1 ), or the distribution of anisotropy fields (fig. 3) in $\mathrm{M}-1$, either a distribution in chain length (model $\mathrm{A}$ only), or a distribution of $\beta$ (model $\mathrm{A}$ or $\mathrm{A}^{\prime}$ ), or both, must be assumed. Finally, it is interesting to note that the acicular magnetites had significantly higher values of coercivity than M-1 (see table 4), even though the particle dimensions were comparable. This disagreement may partly reflect the finite separation of magnetosomes along the chain length. This result may also indicate a completely different reversal mechanisms for acicular particles as suggested by Knowles [46].

\subsection{Implications for paleomagnetism}

Despite the successes of paleomagnetism, the mechanisms by which the remanent magnetization of marine sediments is acquired, and subsequently 
retained over geologic time remains poorly understood. Rock magnetic studies indicate that the remanence in many marine sediments reside in SD-like particles of magnetite (e.g., ref. [47]). Yet, the exact identification of these particles, in many cases, has not been made. Since the discovery of magnetotactic bacteria, it has been suggested that fossil biogenic SD magnetite may be the primary carrier of remanent magnetization in marine sediment $[6-10]$.

Petersen et al. [8] proposed some simple magnetic measurements to demonstrate the existence of biogenic magnetite in deep-sea sediments. However, our results, using the same measurements, are not consistent with those in ref. [8]. There are several possible explanations for this discrepancy. First, the parameters used in ref. [8] are likely to distinguish between any type of SD from MD particle, and not just biogenic magnetite. Second, we do not know if different species, or ancient species. of magnetotactic bacteria would exhibit slightly different magnetic properties. Third, the fossil biogenic magnetite could conceivably act as individual particles, whereas in our samples they are still in chains. For all these reasons, it is not too surprising that our results differ significantly from those in ref. [8].

The SD nature of magnetosomes is clearly demonstrated. The problem is how the magnetosomes are incorporated into the sediments. Do the chains remain intact, or do the individual particles separate and then agglomerate? Preliminary results [7-10] suggests that the chains remain intact after deposition. If so, the marked contrast between acquisition and demagnetization of SIRM (fig. 8) for $\mathrm{M}-1$ and $\mathrm{M}-2$ would suggest a simple magnetic test for the presence of magnetosome chains. The unique type II behavior exhibited by M-1 would be diagnostic for intact magnetosomes. However, several factors could serve to mask the type II behavior. As far as we know, all non-biogenic magnetic phases in rocks exhibit type I behavior. Therefore, the presence of any non-biogenic magnetite or any other magnetic phases, in sufficient quantity, could dominate the SIRM behavior. Finally, our results suggest that until a type II response is observed in marine sediments, electron microscope observation of magnetite morpholo- gies is the only unambiguous technique for distinguishing between lithogenic and biogenic magnetite.

\section{Conclusions}

(1) Freeze-dried powders of A. magnetotacticum containing either intact whole cells (M-1) or magnetosome chains separated from cells (M-2) exhibited single-domain behavior.

(2) An average magnetic dipole moment per cell of $2.4 \times 10^{-11} \mathrm{Am}^{2}$ was determined by magnetically induced birefringence. The average magnetic moment corresponded to about 10 magnetosomes per cell.

(3) The acquisition and demagnetization of IRM and ARM and the time dependence of VRM were significantly different between $\mathrm{M}-1$ and $\mathrm{M}-2$. This contrast in magnetic behavior was attributed to different degrees of particle agglomerations or. equivalently, to differences in the strength of the interaction fields in each sample. Particle agglomeration was greater and interaction fields larger in M-2 because the extracted magnetosomes chains were no longer separated from one another by the cell membranes and cytoplasm of the bacteria.

(4) AF demagnetization spectrum of SIRM was shifted towards higher fields with respect to the dc spectra for M-1, whereas the opposite behavior was observed for M-2. According to the classification scheme of Kneller [26]; M-1 and M-2 exhibited type II and type I behavior, respectively. The reason for this difference, although qualitatively related to magnetostatic interactions, was unclear.

(5) M-1 exhibited magnetic properties that were markedly different from those in synthetic dispersed powders of comparable grain size.

(6) The chain-of-spheres model predicted values for the coercive force, the rotational hysteresis parameter, and the ratio $H_{c}$ (random) $/ H_{c}$ (aligned) that agreed with experimental values for $\mathrm{M}-1$. This model was consistent with unique linear arrangement of equidimensional single-domain particles in A. magnetotacticum. 
(7) The unique type II behavior of M-1 would suggest a simple magnetic method for determining the presence of intact magnetosome chains in sediments, although several factors could mask this effect in natural samples.

\section{Acknowledgements}

This research was partially supported by DARPA through Biomagnetech Corporation. We thank Y. Gorby and N. Blakemore for sample preparation, C.R. Rosenblatt for birefringence measurements and Naoma Doherty for remanence measurements. RBF was partially supported by the Office of Naval Research.

\section{References}

[1] R.P. Blakemore, Ann. Rev. Microbiol. 36 (1982) 217.

[2] R.B. Frankel, Ann. Rev. Biophys. Bioeng. 13 (1984) 85.

[3] D.L. Balkwill, D. Maratea and R.P. Blakemore, J. Bacteriol. 141 (1980) 1399.

[4] S. Mann, R.B. Frankel and R.P. Blakemore, Nature 310 (1984) 405.

[5] R.B. Frankel and R.P. Blakemore, J. Magn. Magn. Mat. $15-18$ (1980) 1562.

[6] J.L. Kirschvink and H. Lowenstam, Earth Planet. Sci. Lett. 44 (1979) 193.

[7] S.B.R. Chang, J.L. Kirschvink and J.F. Stoltz, Phys. Earth. Planet. Inter. 46 (1987) 289

[8] N. Petersen, T. von Dobeneck and H. Vali, Nature 320 (1986) 611

[9] J.L. Kirschvink and S.R. Chang, Geology 12 (1984) 559.

[10] J.F. Stolz, S.R. Chang and J.L. Kirschvink, Nature 321 (1986) 849.

[11] R.P. Blakemore, D. Maratea and R.S. Wolfe, J. Bacteriol. 140 (1979) 720.

[12] Y. Gorby, T.J. Beveridge and R.P. Blakemore, J. Bacteriol. 170 (1988) in press.

[13] R.P. Blakemore, K.A. Short, D.A. Bazylinski, C. Rosenblatt and R.B. Frankel, Geomicrobiol. J. 4 (1985) 53.

[14] C. Rosenblatt, F.F.T. de Araujo and R.B. Frankel, J. Appl. Phys. 53 (1982) 2727.

[15] C. Rosenblatt, F.F.T. de Araujo and R.B. Frankel, Biophys. J. 40 (1982) 83.
[16] C.R. Denham, R.P. Blackemore and R.B. Frankel, IEEE Trans. Magn. MAG-16 (1980) 1006.

[17] E.C. Stoner and W.F. Wohlfarth, Trans. Roy. Soc. (London) A240 (1948) 599.

[18] I.S. Jacobs and C.P. Bean, Phys. Rev. 100 (1955) 1060.

[19] P.J. Flanders, J. Appl. Phys. (1988) in press.

[20] P.J. Flanders, IEEE Trans. Magn. MAG-21 (1985) 1584.

[21] D. Dunlop, Farth Planet. Sci. Lett. 78 (1986) 288.

[22] E.P. Wohlfarth. J. Appl. Phys. 29 (1958) 595

[23] S. Cisowski, Phys. Earth Planet. Inter. 26 (1981) 56.

[24] P. Dankers, Geophys. J. Roy. Astron. Soc. 64 (1981) 447.

[25] G.W.D. Spratt, P.R. Bissell and R.W. Chantrell, IEEE Trans. Magn. MAG-22 (1986) 659.

[26] E. Kneller, in: Magnetism and Metallurgy, eds. A.E. Berkowitz and E. Kneller (Academic Press, New York, 1969) p. 366.

[27] D.J. Dunlop and G.F. West, Rev. Geophys. Space Phys. 7 (1969) 709

[28] E. Schmidbauer and N. Schembera, Phys. Earth Planet. Inter. 46 (1987) 77.

[29] W. Lowrie and M. Fuller, I. Geophys. Res. 76 (1971) 6339.

[30] M.E. Bailey and D.J. Dunlop, Earth Planet. Sci. Lett. 63 (1983) 335

[31] W.F. Jaep, J. Appl. Phys. 42 (1971) 2790.

[32] S.K. Banerjee and J.P. Mellema, Earth Planet. Sci. Lett. 23 (1974) 177

[33] R.W. Chantrell and E.P. Wohlfarth, J. Magn. Magn. Mat. 40 (1983) 1.

[34] R. Pauthenet, C.R. Acad. Sci. (1950) 1842.

[35] L. Néel, Ann. Geophys. 5 (1949) 99.

[36] D.J. Dunlop, Geophys. J. Roy. Astron. Soc. 74 (1983) 667.

[37] B.M. Moskowitz, Geophys. J. Roy. Astron. Soc. 82 (1985) 143.

[38] R.V. Chamberlin, J. Appl. Phys. 57 (1985) 3377.

[39] D. Walton and D.J. Dunlop, Solid State Commun. 53 (1985) 359

[40] R.H. Chantrell, M. Fearon and E.P. Wohlfarth, Phys. Stat. Sol. (a) 97 (1986) 213.

[41] R. Thompson and F. Oldfield, Environmental Magnetism (Allen and Unwin, London, 1986) p. 227.

[42] D.J. Dunlop, J. Geophys. Res. 78 (1973) 7602.

[43] D.J. Dunlop, M.E. Bailey and M.F. Westcott-Lewis, Geochim. Cosmochim. Acta 39 (Suppl. 6) (1975) 3063.

[44] J.W. King, S.K. Banerjee and J. Marvin, J. Geophys. Res. 88 (1983) 5911.

[45] D.J. Dunlop, J. Geophys. Res. 91 (1986) 9569.

[46] J.E. Knowles, IEEE Trans. Mag. MAG-20 (1984) 84.

[47] R. Freeman, Geophys. J. Roy. Astr. Soc. 85 (1986) 433. 